\title{
Evaluación de competencias en salud: elaboración y validación de un cuestionario, primeros resultados
}

\section{Skills assessment in health care: the development and validation of a questionnaire, early findings}

\author{
Valentín Gavidia Catalán ${ }^{1}$ \\ http:/ / orcid.org/0000-0001-9153-147X \\ Marta Talavera Ortega ${ }^{1}$ \\ https://orcid.org/0000-0002-1250-0200 \\ Oscar Raúl Lozano Lucía ${ }^{1}$ \\ http://orcid.org/0000-0002-2883-5371
}

\begin{abstract}
Resumen: El objetivo de este trabajo ha consistido en elaborar y validar una herramienta en forma de cuestionario con la que averiguar las competencias en salud que posee el alumnado al finalizar la educación obligatoria. Su importancia radica en que sirve para conocer el aprendizaje producido en Educación para la Salud durante ese tiempo y, por tanto, es punto de referencia para la actuación educativa posterior. El cuestionario trata 24 problemas significativos para la salud, ha sido sometido a juicio de expertos, evaluado con una prueba de legibilidad, con entrevistas a la población diana, y con una prueba piloto con alumnos de $1^{\circ}$ y $4^{\circ}$ de la Educación Secundaria Obligatoria (12 y 16 años). Sus resultados sirven para detectar carencias y las cuestiones invitan a utilizar las metodologías didácticas adecuadas para que todos los estudiantes tengan la oportunidad de mejorar su formación en salud y desarrollar las competencias que permitan mejorar la calidad de vida personal, colectiva y ambiental.
\end{abstract}

Palabras clave: Educación para la salud. Educación secundaria obligatoria. Cuestionario. Competencia en salud.

\begin{abstract}
The aim of this study is to develop and validate a self-administered written questionnaire. It will be a tool to find out the health-care skills that students will have reached at the end of their compulsory education stage. Its significance lies in the usefulness of knowing the Health Education learning achieved during that time and, therefore, as a reference point for subsequent teaching interventions. The questionnaire has been designed to include 24 significant problems for the students' health. It has been subjected to expert opinion, and has been evaluated with a readability test, interviews with the target population, and has been piloted with students of 1 st and 4 th years of secondary education (12 and 16 years old). The questionnaire results serve to identify gaps, and the questions in it encourage the use of proper teaching methodologies, which give students the opportunity to improve their health-care education training and to develop skills to enhance the quality of their lives at the personal, collective and environmental domains.
\end{abstract}

Keywords: Health education. Compulsory secondary education. Questionnaire. Health literacy.

\footnotetext{
${ }^{1}$ Universidad de Valencia, Departamento de Didáctica y Ciencias Experimentales y Sociales, Valencia, España. E-mail: valentin.gavidia@uv.es
} 


\section{Introducción}

Las Competencias básicas o claves del currículo escolar obligatorio español (ESPAÑA, 2006, 2015) permiten poner el acento en aquellos aprendizajes que se consideran imprescindibles, con un planteamiento integrador, orientado a la aplicación de los saberes adquiridos y que deben haberse desarrollado al finalizar la enseñanza obligatoria para alcanzar la realización personal, ejercer la ciudadanía activa, incorporarse a la vida adulta de manera satisfactoria y ser capaz de desarrollar un aprendizaje permanente a lo largo de la vida (LÓPEZ MUÑOZ, 2006). Siguen las indicaciones del Consejo de la Unión Europea (2004) de reformar los sistemas educativos y adaptarlos a una sociedad del conocimiento.

Son siete las competencias clave en el actual currículo y de entre ellas, dos son las que se encuentran directamente relacionadas con la Educación para la Salud: la competencia matemática y en ciencia y tecnología, y la competencia social y cívica. La primera considera de manera muy específica la salud, ya que pretende que el alumnado incorpore "[...] habilidades para desenvolverse adecuadamente, con autonomía e iniciativa personal en ámbitos de la vida y del conocimiento muy diversos (salud, actividad productiva, consumo, ciencia, procesos tecnológicos, etc.)" (ESPAÑA, 2007, p. 687). En lo relacionado con la salud indica que:

[...] partiendo del conocimiento del cuerpo humano, de la naturaleza y de la interacción de los hombres y mujeres con ella, permite argumentar racionalmente las consecuencias de unos u otros modos de vida, y adoptar una disposición a una vida física y mental saludable en un entorno natural y social también saludable. Asimismo, supone considerar la doble dimensión individual y colectiva - de la salud, y mostrar actitudes de responsabilidad y respeto hacia los demás y hacia uno mismo. (ESPAÑA, 2007, p. 687).

Por otro lado, la competencia social y cívica incorpora formas de comportamiento individual que capacitan a las personas para convivir en una sociedad cada vez más plural, relacionarse con los demás, cooperar, comprometerse y afrontar los conflictos. Supone ser capaz de ponerse en el lugar del otro, aceptar las diferencias, ser tolerante y respetar los valores, las creencias, las culturas y la historia personal y colectiva de los otros. (ESPAÑA, 2007).

Teniendo en cuenta la obligatoriedad del desarrollo de estas dos competencias, la necesidad de llevar a cabo la alfabetización en salud (EUROPEAN CANCER ORGANIZATION, 1990), y reconociendo la inexistencia de un currículo específico de salud, resulta necesario tratar de conocer el grado de adquisición de las competencias en salud del alumnado a su paso por la Educación Obligatoria (etapa primaria y etapa secundaria obligatoria). Por ello, nos planteamos como objetivo en el presente trabajo elaborar una herramienta, en forma de cuestionario, con la que conocer las competencias en salud que posee el alumnado al finalizar su escolarización obligatoria y la diferencia existente entre las dos etapas, que nos marcará, en parte, la eficacia de la escolarización en el desarrollo competencial pues la información, habilidades y actitudes hacia la problemática de la salud no se obtiene exclusivamente en la escuela sino que también intervienen otros agentes como la familia, amigos, medios de comunicación, etc. Al mismo tiempo adelantamos los primeros datos obtenidos en la puesta a punto de dicho 
cuestionario. Estos resultados, extraídos del análisis de las respuestas, deben señalar al profesorado los esfuerzos a realizar en la adquisición de las competencias en salud por su alumnado.

\section{Antecedentes y fundamentación teórica}

En nuestro objetivo de definir y evaluar competencias en salud debemos tener en cuenta tres ideas: (a) la concepción de salud; (b) la alfabetización en salud en el contexto de la Educación para la Salud; y (c) las tres dimensiones que poseen las competencias en salud.

a) El concepto de salud. La idea de salud es dinámica, cambia con la época, la cultura y las condiciones de vida y está limitada por el marco social (SAN MARTIN, 1981). Seguiremos, de forma muy resumida, la evolución que ha sufrido en los últimos tiempos, adjudicando a cada una de las concepciones un determinado nivel de representación en función de su complejidad (GAVIDIA, 1998):

- Nivel I. Visión tradicional: entender la salud como la ausencia de enfermedades e invalideces. Es la condición normal de vida y lo que la altera es la enfermedad, de manera que lo importante es vivir sin observar nada extraño, dado que sólo cuando algo no funcionan bien es cuando nos damos cuenta de ello (LERICHE, 1937). Este nivel posee una dimensión estrictamente física.

- Nivel II. Visión preventiva: la Organización Mundial de la Salud (2014, p. 1) en su Carta Constitucional presenta la salud como "[...] el estado de completo bienestar físico, mental y social y no solamente la ausencia de afecciones o enfermedades". Esta definición se realiza en términos positivos e incluye, además de la dimensión física anterior, la psíquica y social.

- Nivel III. Visión ambientalista: Dubos (1959) indica que la salud es el estado de adaptación al medio y la capacidad de funcionar en las mejores condiciones en dicho medio. Se entiende la salud como el estado de equilibrio entre el individuo y su entorno, de forma que cuando éste se altera se produce la enfermedad. El medio ambiente se incorpora a la concepción de salud y el locus control es externo ya que el ambiente determina la calidad de vida.

- Nivel IV. Visión de desarrollo personal: el X Congreso de Médicos y Biólogos de Lengua Catalana celebrado en Perpiñán en 1976 señala que la salud es aquella manera de vivir que es autónoma, solidaria y profundamente gozosa. Sitúa al individuo como protagonista de su propia salud, puesto que es responsable de las acciones que pueden quebrantarla o mejorarla. El locus control es interno.

- Nivel V. Visión de desarrollo social: el concepto de salud de la Oficina Regional para Europa de la OMS (WORLD HEALTH ORGANIZATION, 1984, p. 2, traducción nuestra) indica que "La salud es la capacidad de realizar el propio potencial personal y responder de forma positiva a los retos del ambiente". Señala la importancia del desarrollo de todas las capacidades individuales, pero dirigidas a transformar el entorno haciéndolo más humano y saludable. Se concibe la salud holística o global, como proceso multicausal que se identifica con el desarrollo personal y social y como medio para la mejora de la calidad de vida. 
b) La alfabetización en salud en el contexto de la Educación para la Salud. En la sociedad en la que vivimos del conocimiento y de la comunicación, cada vez se hace más imprescindible que encontremos nuevas formas de pensar, actuar y sentir ante la complejidad de los problemas que nos acucian. De ahí la urgencia de la alfabetización, que no solo consiste en que las personas reconozcan las letras y sepan leer, sino que sean capaces de usarlas para elaborar textos y comunicarse con los demás. Esta necesidad de alfabetizase no se ha circunscrito únicamente al campo de las letras, sino que se ha extendido a otras áreas y así hablamos de la alfabetización científica (DEBOER, 2000; HODSON, 1988), alfabetización audiovisual (APARICI; GARCÍA-MATILLA, 1998), alfabetización digital (TRAVIESO; PLANELLA, 2008), y también alfabetización en salud (FALCÓN ROMERO; RUIZ-CABELLO, 2012).

La alfabetización para la salud (health literacy) es un concepto complejo introducido en los años 1970 en el mundo anglosajón, que cada día gana mayor reconocimiento en la comunidad científica e indica la dirección a seguir en la Educación para la Salud. La Organización Mundial de la Salud (OMS), en su glosario de términos de salud pública, la define como “[...] las habilidades cognitivas y sociales que determinan la motivación y la capacidad de los individuos para acceder a la información, compreenderla y utilizarla, para promover y mantener una buena salud” (ORGANIZACIÓN MUNDIAL DE LA SALUD, 1998, p. 21).

Para Kickbusch (2008) un individuo con un nivel adecuado de alfabetización en salud tiene las competencias necesarias para gestionar su salud de una forma responsable. Se trata de una exigencia cívica y ética. Cívica en la medida en que la mayoría de los países desarrollados tiene unos sistemas de salud diseñados para ciudadanos responsables; pero también ética ya que el cuidado de uno mismo es una obligación moral en una sociedad que consume cada vez más recursos sanitarios (FALCÓN ROMERO; RUIZ-CABELLO, 2012).

c) Competencias en salud. Este concepto con frecuencia lo encontramos relacionado con habilidad, destreza, capacidad, aptitud, etc., surgidos al amparo de la necesidad de evaluar rendimientos. En concreto, la Organización para la Cooperación y el Desarrollo Económicos (OCDE) define a una persona competente como aquella que tiene los conocimientos, destrezas, actitudes y la conducta profesional indispensables para demostrar satisfactoriamente el desempeño de una tarea, una acción o una función concreta en el ámbito laboral (RYCHEN et al., 2006).

En el caso educativo aparecen con el propósito de superar la tradicional formación en conocimientos disciplinares para adentrarse en una formación más práctica y funcional, que enseñe a saber qué hacer en contextos problemáticos diversos (MARTÍNEZ CLARES; ECHEVARRÍA SAMANES, 2009). La competencia convierte en real la capacidad de movilizar recursos para hacer frente a situaciones o problemas existentes y emergentes y, por lo tanto, impredecibles e indefinidos.

El enfoque de competencias en educación cobra sentido y surge como una solución óptima ante los desafíos que depara la sociedad que demanda "[...] movilizar conocimientos para resolver problemas de forma autónoma, creativa y adaptada al contexto" (MANZANARES; SANCHEZ SANTAMARÍA, 2012, p. 188). Podemos decir, con Delors (1996), Hipkins (2006), Pérez Gómez (2007) y Rychen et al. (2006), que los rasgos diferenciales de las competencias en el mundo educativo son: el constituir un saber hacer complejo y adaptativo para resolver los problemas que se presentan, esto es, un saber que no se aplica de forma mecánica sino reflexiva, susceptible de adecuarse a una diversidad de contextos y que tiene 
carácter integrador, abarcando conocimientos, habilidades, emociones, valores y actitudes. En definitiva, toda competencia incluye un saber, un saber hacer y un querer hacer en contextos y situaciones concretos en función de propósitos deseados. Así, hablamos de competencia como un todo con significado práctico, que implica conocimiento en la acción, conocimiento para la acción y conocimiento sobre la acción, coincidiendo con el concepto de profesional reflexivo definido por Schön (1992).

Muchas veces, la definición de competencias se utiliza como capacidad para realizar de manera adecuada una tarea concreta sin discriminar entre capacidad y competencia y apareciendo ambos términos como sinónimos. El aspecto actitudinal es el que confiere identidad diferenciadora a la competencia, el querer hacer es esencial, y así hablaremos de competencia como la capacidad para realizar de manera adecuada una tarea concreta con la predisposición de hacerla.

Adentrándonos en el campo de la salud, las competencias en salud presentan una especificidad que las distingue del resto, dado que guardan relación con estilos de vida y con la capacidad de responder adecuadamente a problemas o situaciones vitales desde una dimensión tanto personal como social. Hacemos énfasis en los ángulos siguientes: (a) adquisición de capacidades personales; (b) desarrollo de las habilidades con vertiente social; (c) utilidad para hacer frente a los actuales desafíos o problemas de salud; (d) protagonismo del entorno; y, (e) relevancia del aspecto actitudinal, o predisposición a hacer.

Así pues, concebimos la Competencia en Salud como la capacidad y la determinación de resolver los problemas relacionados con la salud personal y colectiva, utilizando recursos (habilidades, conocimientos, experiencias, conductas, etc.) para resolver de forma adecuada un problema de salud en un contexto definido y contribuir a crear un entorno en el que las opciones saludables sean más fáciles de tomar (GAVIDIA, 2016; GAVIDIA et al., 2012).

Podría parecer que los conceptos de alfabetización y desarrollo de competencias son equivalentes y que podría usarse de forma indistinta. Sin embargo, por lo expuesto comprobamos que las competencias añaden unos rasgos distintos y complementarios a la alfabetización, como son la vertiente práctica, la contextual, la problematizadora y la evaluativa.

\section{Metodología}

Para la elaboración y validación de un cuestionario que sirva para evaluar las competencias en salud del alumnado se ha seguido el proceso siguiente.

\section{Elaboración del cuestionario}

Propuesta de cuestionario. Tres miembros del equipo de investigación elaboraron un primer borrador de cuestionario con 4 grandes apartados atendiendo a lo que se pretende evaluar y a las variables a tener en cuenta: (a) datos personales y demográficos; (b) concepto de salud; (c) identificación por parte del alumnado de problemas relacionados con la salud; y, (d) posibles actuaciones ante diferentes problemas de salud. En la selección de problemas se atendió a las indicaciones de la Organización Mundial de la Salud (2010), de la Unión Internacional de Promoción y Educación para la Salud (2004) y del Ministerio Español de 
Sanidad y Consumo (ESPAÑA, 2017). Para la elección de las preguntas se procuró que trataran temas cercanos al alumnado, presentados de forma directa, sencilla y contextualizada, ofreciendo situaciones cotidianas, y atendiendo a los rasgos distintivos de las competencias. En su redacción se tuvo en cuenta las reglas propuestas en la bibliografía (BLANCHET, 1989; MARTÍNEZ, 2003), procurando que fueran sencillas y lo más cortas posibles, directas y relacionadas con el tema, redactadas de forma personal, expresadas en forma neutral o en positivo, nunca negativo o que pudieran levantar prejuicios, teniendo en cuenta al encuestado, sus conocimientos, edad, etc.

Evaluación de legibilidad (nivel de comprensión lectora necesario para entender el contenido). Para ello, se aplicó el programa informático INFLESZ ${ }^{2}$, de acceso libre, que realiza un análisis básico sobre el número de sílabas, el número de palabras, el número de frases, el promedio de sílabas/palabra, el promedio de palabras/frase, la fórmula de perspicuidad y un grado de legibilidad de acuerdo con una escala. Está diseñado para la lengua castellana, es de uso sencillo y ha sido validado. Esta escala INFLESZ posee una puntuación de 0 hasta más de 80, en cinco tramos, desde muy difícil hasta muy fácil (OTERO et al., 2012) (Tabla 1).

Tabla 1 - Grados de legibilidad en la Escala INFLESZ

\begin{tabular}{lll}
\hline \multicolumn{1}{c}{ Puntos } & Nivel del nivel educativo requerido & \multicolumn{1}{c}{ Tipo de publicación } \\
\hline $\mathbf{0 - 4 0}$ & Muy difícil / educación superior & Especializada (universitaria, científica) \\
$\mathbf{4 1 - 5 5}$ & Algo difícil/Educación media superior. & Divulgación científica, prensa especializada \\
$\mathbf{5 6 - 6 5}$ & Normal / Educación media básica. & Prensa general, prensa deportiva \\
$\mathbf{6 6 - 8 0}$ & Bastante fácil / Educación básica & Textos docentes, Novelas exitosas \\
$\mathbf{7 8 0}$ & Muy fácil/ Educación básica & Historietas, cómics. \\
\hline
\end{tabular}

Fuente: Otero et al. (2012).

Pase del cuestionario a una pequeña muestra del alumnado y entrevista posterior. La pequeña muestra estaba formada por 8 alumnos/as, a los que se les ha presentado el cuestionario y se ha tenido una entrevista posterior sobre el mismo. Se pretendía comprobar su compresión, el tiempo necesario para su respuesta, las impresiones que les producían las preguntas y la estética del cuestionario, pues somos conscientes que el diseño desempeña un importante papel en la solución del cuestionario (GOLDIN-MEADOW, 2006). Tanto el pase de los cuestionarios como las entrevistas han sido realizados con consentimiento informado.

\section{Validación del cuestionario}

El cuestionario fue analizado en cuanto a la validez de su contenido mediante un estudio de expertos, y en cuanto a la confiabilidad a través de una prueba piloto calculando el coeficiente Alfa de Cronbach.

\footnotetext{
${ }^{2}$ Recuperado de: https://legibilidad.blogspot.com/2015/01/el-programa-inflesz.html. Accedido en: 9 abr. 2019.
} 
Para el estudio de validez, el grupo de expertos ha estado formado por el equipo de investigación y el grupo de trabajo del proyecto Competencias en Salud (COMSAL), constituido por 29 personas. El equipo de investigación está compuesto por 10 doctores pertenecientes a Universidades españolas (País Vasco, Zaragoza, Las Palmas de Gran Canarias y Valencia). El grupo de trabajo está compuesto por 19 profesores/as, de los cuales 10 son docentes de Educación Primaria o Secundaria de Valencia, 2 de la Universidad de Fortaleza (Brasil), 4 de Institutos de Formación de Profesorado de Buenos Aires y 3 de la Universidad de Neuquén (Argentina), todos ellos con experiencia en la Educación para la salud y en el diseño, elaboración y trabajo con cuestionarios.

Este estudio se llevó a cabo mediante una reunión de un día de duración, en la que se buscaron consensos y se reescribieron algunos enunciados a tenor de los comentarios del alumnado, de los resultados de sus respuestas, y de las opiniones y sensibilidades de los presentes. Al cuestionario reelaborado se le volvió a aplicar el cuestionario de legibilidad INFLESZ.

Prueba piloto. Se aplicó el cuestionario a una muestra constituida por 29 alumnos de $1^{\text {er }}$ curso de la Educación Secundaria Obligatoria (ESO) y 37 alumnos de $4^{\circ}$ de la ESO de un Instituto de Enseñanza Secundaria de la Comunidad Valenciana. Se eligieron estos cursos por ser el primero y último de la ESO y así conocer las competencias adquiridas durante la etapa de Educación Primaria y de la Secundaria Obligatoria. Se buscó el grado de consistencia interna aplicando a los resultados el coeficiente Alfa de Cronbach.

\section{Resultados}

Diseño del cuestionario. El cuestionario está formado por cuatro apartados:

a) Datos personales: donde se tiene en cuenta la edad, el sexo y el centro de estudio.

b) Concepto de salud: se pide escribir al menos cinco palabras relacionadas con la idea de salud. Se evalúa atendiendo a los cinco niveles señalados en la fundamentación teórica: la visión tradicional de ausencia de enfermedad, la visión de bienestar, la de adaptación al entorno, la de conducta y desarrollo personal, y la de atención social (GAVIDIA, 1998).

c) Identificación de problemas de salud: se presentan siete ámbitos de salud y se solicita que señalen problemas relacionados con cada uno de ellos, así como su mayor fuente de información en cada uno de los ámbitos.

d) Actuaciones ante problemas de salud: se eligieron 24 problemas de salud relacionados con los siete ámbitos (Cuadro 1). Cada problema está constituido por tres preguntas diferentes para averiguar si (i) conoce el problema; (ii) sabe qué hacer ante él; y, (iii) es capaz de expresar una opinión o señala una predisposición de actuación, es decir, se realizan tres preguntas diferentes ante una misma situación que tratan de conocer las tres dimensiones que se posee de una competencia: conocimiento, habilidades y predisposición. El equilibrio entre las tres dimensiones nos indicará la coherencia interna de la competencia de quien responde. Por ello, las preguntas que se presentan son abiertas posibilitando la respuesta reflexiva y opcional del alumnado. 
Gavida, V.; Talavera, M.; Lozano, O.R.

Cuadro 1 - Ámbitos de Salud y Problemas asociados que trata el cuestionario

\begin{tabular}{|c|c|c|c|}
\hline $\begin{array}{l}\text { Ámbito de } \\
\text { salud }\end{array}$ & Problemas de salud & $\begin{array}{l}\text { Ámbito de } \\
\text { salud }\end{array}$ & Problemas de salud \\
\hline \multirow[t]{2}{*}{$\begin{array}{l}\text { Alimentación } \\
\text { y Actividad } \\
\text { Física }\end{array}$} & \multirow{2}{*}{$\begin{array}{l}\text { 4- Malnutrición- } \\
\text { Adelgazamiento } \\
\text { 5- Celiaquía } \\
\text { 6- Despilfarro de comida/ } \\
\text { Solidaridad } \\
\text { 7- Actividad física } \\
\text { 20- Higiene postural }\end{array}$} & $\begin{array}{l}\text { Salud mental y } \\
\text { emocional }\end{array}$ & $\begin{array}{l}\text { 1- Ansiedad } \\
\text { 2- Depresión } \\
\text { 3- Violencia y Malos tratos }\end{array}$ \\
\hline & & \multirow[t]{2}{*}{$\begin{array}{l}\text { Vida afectivo } \\
\text { sexual }\end{array}$} & $\begin{array}{l}\text { 8- Sexualidad/Identidad } \\
\text { sexual } \\
\text { 9- Infecciones de transmisión } \\
\text { sexual }\end{array}$ \\
\hline \multirow{3}{*}{$\begin{array}{l}\text { Higiene } \\
\text { personal y } \\
\text { comunitaria }\end{array}$} & \multirow{3}{*}{$\begin{array}{l}\text { 16- Higiene dental: chucherías } \\
\text { y golosinas } \\
\text { 17- Higiene de los vestidos } \\
\text { 18- Higiene corporal } \\
\text { 19- Alergias } \\
\text { 21- Enfermedades infecciosas }\end{array}$} & & 10- Pérdida de la regla \\
\hline & & $\begin{array}{l}\text { Salud } \\
\text { ambiental }\end{array}$ & $\begin{array}{l}\text { 11- Contaminación de las } \\
\text { ciudades } \\
\text { 12- Comercio justo } \\
\text { 13- Cambio climático }\end{array}$ \\
\hline & & \multirow{2}{*}{$\begin{array}{l}\text { Prevención de } \\
\text { Adicciones }\end{array}$} & \multirow{2}{*}{$\begin{array}{l}\text { 22- Adicciones / Marihuana } \\
\text { 23- Automedicación } \\
\text { 24- Adicciones sin mediar } \\
\text { sustancia }\end{array}$} \\
\hline $\begin{array}{l}\text { Prevención de } \\
\text { Accidentes }\end{array}$ & $\begin{array}{l}\text { 14- Accidentes domésticos } \\
\text { 15- Accidentes de tráfico }\end{array}$ & & \\
\hline
\end{tabular}

Fuente: elaborada por los autores.

Entrevista y pase del cuestionario a una muestra de 8 alumnos. El resultado de esta acción hizo que se eliminaran algunas ilustraciones introducidas y se modificaran otras para que no desviaran la atención del alumnado. El tiempo en contestar el cuestionario fue la duración de una sesión de aula, es decir, de 50 minutos, por lo que, para evitar cansancio en el alumnado, se dividió el cuestionario en dos mitades, modelo A y modelo B. Los dos modelos tratan los siete ámbitos de salud, pero con la mitad de los problemas, de forma que resultan complementarios. Así se redujo el tiempo de cumplimentación del cuestionario a unos 25 minutos.

Nivel de legibilidad del texto. Los análisis realizados (Tabla 2) muestran que tanto el Modelo A como el Modelo B presentan un grado de comprensión bastante fácil en el índice INFLESZ, lo que significa que son comprensibles por estudiantes con una educación media-básica.

Validación del cuestionario a partir de la opinión de expertos. Dada la distinta procedencia del grupo de expertos, y de los destinatarios del cuestionario formado por alumnado de diferentes contextos socioculturales, se tuvo un cuidado especial en los enunciados, se modificaron algunos aspectos iniciales, se evitó el uso de términos que podrían crear confusión, y se mantuvieron los problemas elegidos a testar. Se verificó la adecuación de las preguntas a los objetivos de la investigación, la existencia de estructura, la disposición general equilibrada y armónica, la no reiteración o existencia de preguntas superfluas y que no faltaran preguntas o elemento clave para la investigación. 
Tabla 2 - Resultado al Análisis INFLESZ del Cuestionario

\begin{tabular}{lll}
\hline & Modelo A & Modelo B \\
\hline Sílabas & 1550 & 1441 \\
Palabras & 752 & 675 \\
Frases & 155 & 133 \\
Promedio sílabas/palabra & 2.06 & 2.13 \\
Promedio palabras/frases & 4.85 & 5.08 \\
Índice Flesch-Szigrist & 73.57 & 68.76 \\
Grado escala INFLESZ & Bastante fácil & Bastante fácil \\
\hline
\end{tabular}

Fuente: elaborada por los autores.

Atención importante se le concedió a la forma de evaluar las respuestas dado que la mayoría de las cuestiones se formularon de forma abierta. Por ello se elaboró un modelo consensuado de evaluación con las posibles respuestas a cada ítem, asignando una puntuación de 0 cuando no existiera respuesta o ésta no fuera válida, 1 para respuestas parciales y 2 puntos para las respuestas correctas, que ofrecieran dos o más posibilidades, o que mostraran vertientes personales y colectivas de la salud.

Confiabilidad a través de la Prueba piloto: Para conocer el grado de consistencia interna del cuestionario se valoraron las respuestas del alumnado con puntuaciones de 0 y 1 . Con 0 cuando el estudiante deja en blanco la cuestión o la respuesta carece de sentido y con 1 cuando su contestación tiene relación con lo que se le pregunta (aunque la respuesta no sea correcta), dado que se buscan respuestas coherentes.

El cálculo Alfa de Cronbach a sus respuestas da un valor de 0,91, lo que indica un alto valor de confiabilidad y dota al cuestionario de una fuerte coherencia interna. Se trata, entonces, de un instrumento válido para el objetivo de estudiar las competencias en Salud de los estudiantes. Después se estudiaron cada uno de los ámbitos por separado, obteniendo los resultados que aparecen en la Tabla 3. El cuestionario final sobre Competencias en Salud se presenta en el Apéndice.

Tabla 3 - Confiabilidad de los ámbitos de salud

\begin{tabular}{lcc}
\hline \multicolumn{1}{c}{ Ámbitos de salud } & $\mathbf{N}^{\mathbf{0}}$ problemas $\mathbf{-} \mathbf{n}^{\mathbf{0}}$ ítems & Consistencia interna \\
\hline Salud Mental y Emocional & $3-9$ & 0,85 \\
Alimentación y Act. Física & $5-15$ & 0,90 \\
Vida afectivo-sexual & $3-9$ & 0,77 \\
Salud ambiental & $4-12$ & 0,89 \\
Prevención de Accidentes & $2-6$ & 0,91 \\
Higiene & $4-12$ & 0,87 \\
Prevención de adicciones & $3-9$ & 0,91 \\
\hline
\end{tabular}

Fuente: elaborada por los autores. 
Gavida, V.; Talavera, M.; Lozano, O.R.

\section{Primeros Resultados}

Se han analizado 66 cuestionarios, de los cuales 34 son chicas y 32 chicos. El número de encuestas está en función de poder concluir si existen diferencias significativas entre las variables. Los resultados no son concluyentes debido a la escasa muestra, pero señalan las siguientes tendencias:

Sobre las concepciones de Salud: Los términos utilizados por el alumnado, ubicados en los niveles señalados en la metodología, se muestran en la Tabla 4, en la que se tiene en cuenta la etapa escolar (Primaria 12 años, Secundaria 15 años) y la titularidad del centro (Privado-concertado o público).

Tabla 4 - Número de conceptos usados en cada Nivel de salud

\begin{tabular}{rlllll}
\hline $\begin{array}{r}\text { Nivel de } \\
\text { salud }\end{array}$ & Privado 12-13 & Privado 15-16 & Público 12-13 & Público 15-16 & Total \\
\hline N-1 & 9 & 15 & 17 & 12 & 53 \\
N-2 & 1 & 14 & 11 & 16 & 42 \\
N-3 & 1 & 4 & 1 & 1 & 7 \\
N-4 & 17 & 15 & 28 & 10 & 70 \\
N-5 & 1 & --- & -- & --- & 1 \\
\hline
\end{tabular}

Fuente: elaborada por los autores.

En la representación de salud, el alumnado no ha integrado los efectos que produce el entorno ni la idea de mejora de la calidad de vida, es decir, de procurar un entorno físico y social cada vez mejor, incorporando la vertiente colectiva y comunitaria de la salud. Es decir, no se alcanza el nivel 5 de complejidad. Las ideas más arraigadas son las que significan acciones individuales como: actividad física, adecuada alimentación, etc.

Sobre la Alimentación y Actividad física: aparecen bien representados los términos de obesidad, desnutrición y desórdenes alimentarios como la anorexia y la bulimia. Apenas aparece la actividad física y nada sobre las intoxicaciones e infecciones alimentarias.

Sobre las Adicciones: se encuentran bien representadas, por el número de veces que aparece el término, las drogas en general refiriéndose a la marihuana, cocaína, heroína, etc. También el tabaco y el alcohol. No aparecen las adicciones a los medicamentos y aquellas en las que no median sustancias (internet, juegos, etc.)

Sobre la Salud mental: los conceptos que más veces aparecen son la depresión y la tristeza. Apenas se mencionan los desórdenes alimentarios, y nada el tema de la violencia, acoso, baja autoestima y ansiedad. 12 individuos no contestan.

Sobre la Higiene: aparecen términos muy variados y poco repetidos, indicando dispersión de conceptos. Las infecciones es el más repetido y luego hongos, piojos, pulgas, mal olor, caries, sida. No señalan la higiene postural o de los alimentos, las alergias, etc. El número de alumnos que no contestan es el más alto.

Sobre la vida afectiva sexual: el VIH-Sida, que es el concepto que aflora con mayor frecuencia. Luego surgen términos como la sífilis, la violación, embarazos de adolescentes, etc. No señalan los derivados de la diferente identidad sexual y 11 alumnos no contestan. 
Sobre los Accidentes: hay mucha dispersión de conceptos: traumatismos, invalideces, heridas, etc. en general ligadas a los accidentes de tráfico. Escaso eco tienen los accidentes domésticos y ninguno las caídas, las quemaduras, asfixias o mordeduras de animales. El número de no respuestas es alto: 13 .

Sobre la Salud ambiental: la contaminación es el término más repetido. Con menor frecuencia la deforestación, basuras, alergias y problemas respiratorios. Apenas se menciona el ruido, las relativas a las catástrofes naturales, la pérdida de la biodiversidad y las relacionadas con la sostenibilidad. El número de alumnos que no contestan es muy alto: 15.

\section{Discusión y conclusiones}

Se ha elaborado un cuestionario para conocer las competencias en salud adquiridas por el alumnado al finalizar los estudios obligatorios, constituido por preguntas abiertas sobre problemas de salud. Este cuestionario se ha dividido en dos para que el tiempo de respuesta sea menor, pero son complementarios. No obstante, cada uno de ellos, de forma independiente, es válido para conocer el grado de adquisición de competencias en salud. El tiempo necesario para su respuesta es de 25 minutos aproximadamente, y el cuestionario completo de unos 45 minutos.

Se han elegido 24 problemas de salud considerados importantes en esta edad para estudiar hasta qué punto el alumnado ha adquirido competencias para enfrentarse a ellos. Evidentemente, no se presentan todos los posibles problemas de salud para no hacer un cuestionario interminable, ya que solo se pretende averiguar si, en líneas generales, el alumnado es competente en salud. Son preguntas abiertas sobre 24 problemas con tres dimensiones diferentes, que hacen un total de 72 ítems en los que el alumnado puede contestar con libertad y sin ninguna restricción, por lo que, de su análisis tanto cuantitativo como cualitativo, se puede desprender el grado de adquisición de competencia y su coherencia interna al estudiar la relación existente entre las tres dimensiones que la conforman.

En el momento de decidir el tipo de cuestionario se valoraron otras posibilidades como las preguntas cerradas, o las que posibilitan opiniones tipo Likert, pero se optó por preguntas abiertas precisamente porque una competencia es la confluencia de conocimientos, habilidades, actitudes y predisposiciones para dar respuesta a una situación problemática y deseamos que el alumnado las pueda expresar. Esto significa que a la evaluación cuantitativa se le debe sumar la valoración cualitativa que se efectúa analizando la respuesta. A lo largo del texto hemos señalado la forma de evaluar este cuestionario.

En opinión del grupo de expertos consultados, se puede decir que el cuestionario diseñado es un instrumento válido, fiable y validado, que permite identificar las competencias en salud adquiridas por los estudiantes y la posible eficacia de la educación al comparar los datos de dos edades diferentes. Esta validez se apoya tanto en los resultados obtenidos por las respuestas del alumnado, como en el informe elaborado por el grupo de expertos. No obstante, $\mathrm{y}$ atendiendo al menor coeficiente de Alfa de Cronbach obtenido en el ámbito de la vida afectivo sexual y al análisis de las respuestas emitidas por el alumnado, el informe sugirió que la problemática de este ámbito fuera modificada para el alumnado de $1^{\circ}$ de la ESO debido a su menor edad, manteniendo los enunciados para el alumnado de $4^{\circ}$ de la ESO. 
Gallego (2013) hace hincapié en tres aspectos de la salud: la personal, la relacional y la ambiental y las hace corresponder a tres ámbitos de la competencia en salud: el autocuidado, la adaptación social y la atención al entorno. Sin embargo, las tres preguntas que realizamos para cada problema no responden exactamente a esta visión, sino más bien a las tres dimensiones que presenta el proyecto Pisa para la evaluación de las competencias: conocer el problema, sabe qué hacer, y capacidad de expresar opinión o predisposición.

Es en la corrección de cada pregunta cuando nos acercamos a Gallego (2013) al puntuar si la respuesta que ofrece el alumnado atiende a las dimensiones personal, colectiva o ambiental, otorgando un punto si tiene presente una única dimensión y dos puntos si atiende al menos a dos de las dimensiones. Dejamos la puntuación nula cuando no hay respuesta o está completamente equivocada.

La importancia del presente cuestionario radica en que sirve para conocer lo acaecido durante la Educación Obligatoria en el aprendizaje de la Educación para la Salud y la formación competencial, especialmente si se obtienen respuestas al finalizar la Educación Primaria y la Educación Secundaria y se comparan los resultados. Estos son aprovechables como punto de referencia para actuaciones educativas posteriores, pudiendo incidir en las aulas sobre aquellos problemas cuyos datos presenten mayores deficiencias.

El cuestionario no solo señala los problemas de salud sobre los que se poseen carencias, sino que, al estar formado por preguntas abiertas acerca de habilidades, formas de actuar y opiniones referentes a una misma problemática, permite indagar sobre la coherencia interna de la competencia que se posee. Esta coherencia interna, o equilibrio entre las tres dimensiones de la competencia, la podemos relacionar con la funcionalidad o grado de funcionamiento que se posee ante una determinada cuestión.

Este minucioso conocimiento permite al profesorado el uso de actividades de aprendizaje dirigidas a la adquisición de la competencia en general o encaminadas específicamente hacia una dimensión concreta, lo que equivale al uso de metodologías didácticas diferentes a la mera transmisión de conocimientos y pueda abogar por actuaciones colectivas con una problemática social y ambiental.

Así mismo, los resultados de este cuestionario les interesan a las instituciones que elaboran libros de texto y recursos educativos, como editoriales, colectivos docentes, administraciones, etc., ya que pueden conocer las dimensiones de las competencias menos desarrolladas en el alumnado y preparar materiales que subsanen la situación.

\section{Agradecimiento}

El artículo se enmarca en el proyecto Análisis, Diseño y Evaluación de Recursos Educativos para el desarrollo de Competencias en Salud (EDU2013-46664-P), financiado por el Ministerio de Economía y Competitividad español. En esta investigación han participado, además de los autores, 29 expertos a los que se agradece su colaboración.

\section{Referencias}

APARICI, R.; GARCÍA-MATILLA, A. Lectura de imágenes. Madrid: Ediciones de la Torre, 1998. 
BLANCHET, A. Técnicas de investigación en ciencias sociales. Madrid: Narcea, 1989.

CONSEJO DE LA UNIÓN EUROPEA. Educación y formación 2010: urgen las reformas para coronar con éxito la estrategia de Lisboa. Bruselas: Consejo de la Unión Europea, 2004. Recuperado de: http:// register.consilium.europa.eu/doc/ srv?l=ES\&f=ST\%206905\%202004\%20INIT. Visitado en: 12 dic. 2015.

DEBOER, G. B. Scientific literacy: another look at its historical and contemporary meanings and its relationship to science education reform. Journal of Research in Science Teaching, Hoboken, v. 37, n. 6, p. 582-601, 2000.

DELORS, J. La educación encierra un tesoro: informe a la UNESCO de la Comisión Internacional sobre la educación para el siglo XXI. Paris: UNESCO, [1996]. Recuperado de: http://www.unesco.org/education/pdf/DELORS_S.PDF. Visitado en: 8 abr. 2019.

DUBOS, R. J. Mirage of health: utopias, progress and biological change. New York: Harper, 1959.

ESPAÑA. Ley orgánica 2/2006, de 3 de mayo, de educación. Boletín Oficial del Estado, Madrid, n. 106, p. 17158-17207, 4 mayo 2006. Recuperado de: https://www.boe.es/eli/es/ lo/2006/05/03/2/con. Visitado en: 8 Abr. 2019.

ESPAÑA. Real decreto 1105/2014, de 26 de diciembre, por el que se establece el currículo básico de la educación secundaria obligatoria y del bachillerato. Boletín Oficial del

Estado: sec. 1, Madrid, n. 3, p. 169, 3 en. 2015. Recuperado de: https://www.boe.es/boe/ dias/2015/01/03/pdfs/BOE-A-2015-37.pdf. Visitado en: 8 abr. 2019.

ESPAÑA. Real decreto 1631/2006, de 29 de diciembre, por el que se establecen las enseñanzas mínimas correspondientes a la educación secundaria obligatoria. Boletín Oficial del Estado, Madrid, n. 5, p. 677-773, 5 ene. 2007. Recuperado de: http://sid.usal.es/idocs/ F3/LYN10326/3-10326.pdf. Visitado en: 8 Abr. 2019.

ESPAÑA. Ministerio Español de Sanidad y Consumo. Encuesta nacional de salud de España 2017. Recuperado de: https://www.mscbs.gob.es/estadEstudios/estadisticas/ encuestaNacional/encuesta2017.htm. Visitado en: 8 Abr. 2019.

EUROPEAN CANCER ORGANIZATION. Principales conclusiones de la Conferencia Europea sobre Educación para la Salud y Prevención del Cáncer en las Escuelas. Dublín: OMS, 1990.

FALCÓN ROMERO, M.; RUIZ-CABELLO, A. L. Alfabetización en salud: concepto y dimensiones: proyecto europeo de alfabetización en salud. Revista de Comunicación y Salud, Madrid, v. 2, n. 2, p. 91-98, 2012. Recuperado de: https://dialnet.unirioja.es/ descarga/articulo/4500264.pdf. Visitado en: 10 abr. 2019.

GALLEGO, J. Promoción de la salud. In: JORNADA DE EXPERTOS SOBRE COMPETENCIAS DE SALUD PARA O FUTURO, 1., 2013, Valencia. Recuperado de: http://es.slideshare.net/gallegodieguez/promocion-salud-jovenes-valencia. Visitado en: 24 sep. 2016. 
Gavida, V.; Talavera, M.; Lozano, O.R.

GAVIDIA, V. (coord). Los ocho ámbitos de la educación para la salud en la escuela.

Valencia: Tirant Humanidades, 2016. Recuperado de: https://www.uv.es/comsal/pdf/ librocomsal.pdf. Visitado en: 10 abr. 2019.

GAVIDIA, V. Salud, educación y calidad de vida: de cómo las concepciones del profesorado inciden en la salud. Santa Fé de Bogotá: Coop. Editorial Magisterio, 1998.

GAVIDIA, V.; TALAVERA, M.; SENDRA, C.; LLORENTE, E.; GARCÍA DE LA HERA, M.; GOMAR, B. The evaluation of competences in health. In: INTERNATIONAL TECHNOLOGY, EDUCATION AND DEVELOPMENT CONFERENCE, 6., 2012, Valencia. Proceedings [...]. Valencia: International Association of Technology, Education and Development, 2012. p. 166-173.

GOLDIN-MEADOW, S. Talking and thinking with our hands. Current Directions in Psychological Science, Washington, v. 15, n. 1, p. 34-39, 2006. Recuperado de: http://www. jstor.org/stable/20183069. Visitado en: 10 abr. 2019.

HIPKINS, R. The nature on the key competencies: a background paper. Wellington: New Zealand Council for Educational Research, 2006. Recuperado de: https://www.nzcer.org.nz/ system/files/nature-of-k-round-paper.pdf. Visitado en: 9 dic. 2015.

HODSON, D. Toward a philosophically more valid science curriculum. Science Education, Hoboken , v. 72, n. 1, p. 19-40, 1988. DOI: https://doi.org/10.1002/sce.3730720103.

KICKBUSCH, I. Policy innovation for health. New York: Springer, 2008.

LERICHE, R. Pour una pedagogie de la Santé. Paris: Masson, 1937.

LÓPEZ MUÑOZ, M. A. (coord.). Materiales curriculares de salud alimentaria en secundaria. Madrid: Ministerio de Educación y Ciencia, 2006.

MANZANARES, A.; SANCHEZ SANTAMARÍA, J. La dimensión pedagógica de la evaluación por competencias y la promoción del desarrollo profesional en el estudiante universitario. Revista Iberoamericana de Evaluación Educativa, Madrid, v. 5, n. 1, p. 187202, 2012. Recuperado de: http://www.rinace.net/riee/numeros/vol5-num1_e/art13.pdf. Visitado en: 10 abr. 2019.

MARTÍNEZ, V. C. Diseño de encuestas de opinión. Madrid: Ra-Ma, 2003.

MARTÍNEZ CLARES, P.; ECHEVERRÍA SAMANES, B. Formación basada en competencias. Revista de Investigación Educativa, Murcia, v. 27, n. 1, p. 125-147, 2009. Recuperado de: http://www.redalyc.org/pdf/2833/283322804008.pdf. Visitado en: 10 abr. 2019.

MATEOS, J. M. (coord.). Plan de educación para la salud en la escuela: región de Murcia (2005-2010). Murcia: Centro de Recursos de Promoción y Educación para la Salud, 2005.

ORGANIZACIÓN MUNDIAL DE LA SALUD. Adolescentes: riesgos para la salud y soluciones. [2010]. Recuperado de: http://www.who.int/mediacentre/factsheets/fs345/es/ index.html. Visitado en: 9 abr. 2019. 
ORGANIZACIÓN MUNDIAL DE LA SALUD. Competencias básicas en materia de salud y desarrollo de los adolescentes para los proveedores de atención primaria: incluido un instrumento para evaluar el componente de salud y desarrollo de los adolescentes en la formación previa al servicio de los proveedores de atención sanitaria. Ginebra: Organización Mundial de la Salud, 2015.

ORGANIZACIÓN MUNDIAL DE LA SALUD. Constitución. In: DOCUMENTOS básicos. 48. ed. Ginebra: OMS, 2014. Recuperado de: http:/ /apps.who.int/gb/bd/PDF/ bd48/basic-documents-48th-edition-sp.pdf?ua=1\#page=7. Visitado en: 9 abr. 2019.

ORGANIZACIÓN MUNDIAL DE LA SALUD. Fomento de la salud a través de la escuela: informe de un comité de expertos de la OMS en educación sanitaria y fomento de la salud integrales en las escuelas. Ginebra: Organización Mundial de la Salud, 1997.

ORGANIZACIÓN MUNDIAL DE LA SALUD. Promoción de la salud: glosario. Ginebra: OMS, 1998. Recuperado de: https://apps.who.int/iris/handle/10665/67246. Visitado en: 9 abr. 2019.

OTERO, M. J. et al. Cuestionario de autoevaluación de la seguridad de los sistemas automatizados de dispensación de medicamentos. Salamanca: Instituto para el Uso Seguro de los Medicamentos, 2012. Recuperado de: http://www.ismp-espana.org/ficheros/ Cuestionario\%20\%20SAD.pdf. Visitado en: 9 abr. 2019.

PÉREZ GÓMEZ, A. I. La naturaleza de las competencias básicas y sus aplicaciones pedagógicas. Santander: Consejería de Educación de Cantabria, 2007. (Cuadernos de Educación de Cantabria, 1). Recuperado de: https://www.educantabria.es/docs/info_ institucional/publicaciones/2007/Cuadernos_Educacion_1.PDF. Visitado en: 9 abr. 2019.

RAMOS, P. et al. Escuelas saludables y participativas: evaluación de una estrategia de salud pública. Gaceta Sanitaria, Barcelona, v. 27, n. 2, p. 104-110, 2013. DOI: http://dx.doi. org/10.1016/j.gaceta.2012.04.002.

RYCHEN, D. S. et al. (comp.). Las competencias clave para el bienestar personal, social y económico. Málaga: Ediciones Aljibe, 2006.

SAN MARTIN, H. Manual de salud pública y medicina preventiva. Barcelona: Masson, 1981.

SCHÖN, D. A. La formación de profesionales reflexivos. Madrid: Paidos: MEC, 1992.

TRAVIESO, J. L.; PLANELLA, J. La alfabetización digital como factor de inclusión social: una mirada crítica. UOC Papers, Barcelona, n. 6, p. 1-9, 2008. Recuperado de: http:/ /www. uoc.edu/uocpapers/6/dt/esp/travieso_planella.pdf. Visitado en: 9 abr. 2019.

UNIÓN INTERNACIONAL DE PROMOCIÓN Y EDUCACIÓN PARA LA SALUD. Evidencia de la eficacia de la promoción de la salud. Madrid: Ministerio de Sanidad y Consumo, 2004. 
Gavida, V.; Talavera, M.; Lozano, O.R.

WORLD HEALTH ORGANIZATION. Regional Office for Europe. Health promotion: a discussion document on the concept and principles. Copenhagen: WHO Regional Office for Europe, 1984. Recuperado de: http://www.who.int/iris/handle/10665/107835. Visitado en: 9 abr. 2019.

ZABALA, A.; ARNAU, L. Cómo aprender y enseñar competencias: 11 ideas clave. Barcelona: Graó, 2007.

\section{Anexo A - Cuestionario para Evaluar Competencias en Salud (modelo A)}

\section{A. Datos personales}

Género: $\square$ Masculino

Femenino

Edad:

Centro Educativo:

B. Concepto de salud. Escribe al menos cinco palabras relacionadas con tu idea de salud.

C. Señala los problemas de salud que conozcas de cada uno de estos ámbitos. A continuación, indica en cada uno de ellos, cuál ha sido tu fuente de información más importante (la familia, escuela, amigos, internet, cine, documentales, etc.):

Alimentación y Actividad física:

Adicciones:

Vida sexual/afectiva:

Higiene:

Salud mental y emocional:

Accidentes:

Medio Ambiente:

D. Actuaciones ante problemas ¿qué harías en estos casos?

1- Próximamente tienes un examen y piensas que no lo llevas bien preparado. A medida que se acerca te notas más extraño/a

Si esto te ha pasado alguna vez ¿puedes describir cómo te sientes?

¿Qué haces en esta situación?

¿A qué crees que puede ser debido?

4- Últimamente observas tu figura algo más ancha que de costumbre y decides adelgazar... ii4 kilos!! ¿A qué puede ser debido tu aumento de peso?

¿Qué dejarías de comer? ¿En cuánto tiempo te propones lograr tu objetivo?

¿A qué crees que se debe el aumento de sobrepeso/obesidad en la población española?

6- Al terminar el recreo observas en el suelo del patio trozos de pan y bocadillos prácticamente enteros. Sin embargo, sabes que hay niños que pasan hambre y no tienen nada para almorzar.

¿Qué puedes decir sobre el hambre en el mundo?

¿Qué puedes hacer

¿Qué opinas acerca de que estas dos situaciones se produzcan en el mundo actual? 
8- Estás en una fiesta y observas que una persona insinúa con insistencia que quiere mantener relaciones sexuales con la que parece ser su pareja, quien no está de acuerdo, y comienzan a discutir de forma acalorada.

¿Qué problema puede haber detrás de esta situación?

En esta situación ¿qué debe hacer la pareja si no quiere mantener relaciones sexuales?

¿Cómo se podrían combatir situaciones semejantes, tanto personal como socialmente?

9- La tutora quiere que tu clase elabore un informe, con carteles y trípticos, sobre las infecciones de transmisión sexual (ITS) más frecuentes para divulgarlo por el centro educativo.

¿Qué conoces sobre este tipo de infecciones? Anota algún ejemplo

¿Qué mensajes tratarías de presentar?

¿Crees que es un tema importante? ¿Por qué?

12- Desde hace un año tienes un teléfono móvil que funciona a la perfección, sin embargo, acaba de salir al mercado un último modelo que es "lo último en telefonía" y que te gusta mucho.

¿Qué problemas puede acarrear un exceso de consumo?

¿Qué haces con tu antiguo teléfono? ¿Continúas con él o tratas de sustituirlo? ¿Por qué?

¿Qué medidas se podrían adoptar para reducir el consumo en los países desarrollados?

13- La idea de cambio climático está siendo muy utilizada en los medios de comunicación.

¿Cómo se manifiesta este cambio climático? ¿Qué consecuencias podría tener en la salud?

¿Qué puedes hacer para frenar este proceso?

¿Qué opinión te merece las alertas sobre el tema por parte de los científicos y medios de comunicación?

16- Te gusta comer golosinas y chucherías, aunque has oído que no son buenas para la salud.

¿Por qué dicen esto de las golosinas? ¿Qué pueden provocar?

No obstante, cuando las comes ¿haces algo para proteger tu salud?

¿Qué medidas se te ocurren proponer para reducir el consumo excesivo de golosinas?

18- Acabas de terminar de hacer una actividad física (jugar al futbol, clase de pilates, carreras...) en la que has sudado bastante.

¿Qué ocurre si no te lavas/duchas convenientemente?

¿Cuál es tu conducta relativa a la higiene corporal?

Señala las facilidades y las dificultades que encuentras para llevar a cabo una higiene corporal adecuada

20- Durante todo el curso, tu hermano pequeño (10 años) se ha venido quejando de dolores en la espalda. En cambio, durante las vacaciones, no se ha quejado ni un solo día.

¿A qué crees que puede ser debido?

¿Qué le recomendarías hacer?

¿Qué medidas debería adoptar tu familia y la escuela para evitar estos problemas de espalda?

21- En ocasiones, al salir de fiesta observas a algún amigo con un "porro" o "canuto".

¿Qué efectos puede tener esto sobre su salud?

Tus amigos insisten en que te unas a ellos, pero no te apetece fumar ¿qué haces?

¿Qué opinas del consumo de marihuana, tabaco, pastillas...? 
24- Tienes un amigo que está mucho tiempo conectado a las redes sociales y participando en juegos "on line".

¿Crees que puede afectarle a su vida diaria? ¿Cómo?

¿Puedes ayudarle de alguna forma? ¿Cómo?

¿Qué opinión te merecen este tipo de adicciones?

\section{Anexo B - Cuestionario para Evaluar Competencias en Salud (modelo B)}

\section{A. Datos personales}

Género: $\square$ Masculino

Femenino

Edad:

Centro Educativo:

B. Concepto de salud. Escribe al menos cinco palabras relacionadas con tu idea de salud.

C. Señala los problemas de salud que conozcas de cada uno de estos ámbitos. A continuación, indica en cada uno de ellos, cuál ha sido tu fuente de información más importante (la familia, escuela, amigos, internet, cine, documentales, etc.):

Alimentación y Actividad física:

Adicciones:

Vida sexual/afectiva:

Higiene:

Salud mental y emocional:

Accidentes:

Medio Ambiente:

D. Actuaciones ante problemas ¿qué harías en estos casos?

2- Últimamente piensas que uno de tus amigos/as está "depre" o triste.

¿Qué cosas ves en él/ella que te hacen pensar así?

¿Qué puede hacer tu amigo/a para salir de esa situación?

¿Qué se podría hacer para ayudarle?

3- Sobre la violencia y malos tratos...

¿Qué tipos de violencia conoces?

¿Qué podrías hacer en cada uno de los casos que has citado?

¿Por qué es rechazable la violencia? 
5- Uno de los asistentes a una fiesta lleva su comida en un recipiente. Puede tratarse de una persona celíaca, diabética o con intolerancia a la lactosa. Elige una opción y contesta las siguientes preguntas.

$\square$ Celíaco. $\square$ Diabético $\square$ Intolerante a la lactosa

¿Qué problemas puede tener esta persona?

¿Qué alimentos no puede comer?

¿Qué podrías hacer tú y cómo crees que la sociedad debe responder a esta enfermedad?

7- Has notado que algunos adultos cuando hacen alguna actividad como caminar, atarse los zapatos, colgar un cuadro, etc. respiran de manera más acelerada de la normal.

¿A qué crees que puede ser debido?

¿Cómo pueden evitarlo?

¿En qué les puedes ayudar?

10- Una amiga te dice que hace casi dos meses que no ha tenido la regla/menstruación.

¿A qué crees que puede ser debido?

¿Qué le puedes aconsejar?

¿Qué opinas de este tipo de situaciones?

11- La contaminación que sufren las ciudades es un hecho denunciado repetidamente por los medios de comunicación.

¿Qué tipos de contaminación conoces? ¿Qué consecuencias puede tener en los ciudadanos?

¿Qué propones para evitarlo?

¿Crees que es una situación alarmante o están exagerando? ¿Por qué?

14- Es frecuente oír noticias sobre accidentes dentro del hogar....

¿Qué peligros observas en tu casa?

¿Qué puedes hacer para evitar estos peligros?

¿Cómo se podría mejorar la seguridad de las viviendas?

15- Hay gente que le gusta ir en bicicleta y muchas veces la coge para ir a clase, trabajo, de paseo....

¿Qué peligros puede tener el uso de bicicletas en la ciudad?

Si alguien sufre un accidente y se hiciera daño ¿qué harías?

¿Qué propones para evitar estos peligros?

17- Un día muy caluroso de verano uno de tus amigos sugiere un paseo después de comer a pleno sol... ¿Cómo podría afectar esta situación a vuestra salud?

¿Qué precauciones podrías tomar en una ocasión semejante?

¿Qué se te ocurre decirle a tu amigo?

19- Tienes un primo que cuando hacen limpieza en su habitación sale de ella. Dice que tiene "alergia al polvo".

¿Qué significa “alergia al polvo”? ¿Cómo se manifiesta?

¿Qué se podría hacer para minimizar su alergia?

Las autoridades sanitarias dicen que cada vez hay más alérgicos ¿a qué crees que puede ser debido? 
21- Sin darte cuenta, te has hecho un corte no muy profundo pero que sangra. Un/a compañero/a te dice que podrías haberte contagiado del tétanos...

¿Por qué te dice esto?

¿Qué crees que debes hacer en una situación como ésta? ¿Se puede prevenir la enfermedad del tétanos? Algunas, pocas, familias deciden no vacunar a sus hijos ¿qué opinas de esta decisión? Argumenta tu respuesta.

23- Has observado que algún adulto de tu familia, con relativa frecuencia, toma pastillas para dormir y que a los primeros signos de resfriado los combate con antibióticos que guarda en el botiquín.

¿Qué problemas podría causarle esta automedicación?

¿Qué se puede hacer sobre este tema?

¿Qué opinas de la automedicación?

Artigo recebido em 06/03/2018. Aceito em 17/11/2018.

Contacto: Universidad de Valencia, Didactica de las Ciencias

Experimentales y Sociales, Avd. Tarongers, 4, Valencia 46022,

España. 